\title{
La variación en maya yucateco: un estudio descriptivo desde la dialectología perceptual
}

Variation in Yucatec Maya: A descriptive study from perceptual dialectology

\author{
Edith Hernández Méndez \\ Universidad de Quintana Roo, Departamento de Humanidades, \\ División de Estudios Políticos y Humanidades
}

\section{Resumen}

Desde la perspectiva de la dialectología perceptiva y mediante un estudio exploratorio y descriptivo, nos propusimos examinar la habilidad de quince bilingües de maya-español para identificar posibles variedades de lengua maya y relacionarlas con una región geográfica, determinar los rasgos más salientes que usan para distinguir una variedad de otra e identificar algunos factores que pueden afectar esta percepción dialectal. Algunas tareas de la metodología usada por Preston (1999), Mase (1999) y Clopper y Pisoni (2004) fueron adaptadas en el presente estudio, como la tarea de categorización controlada con mapa y la tarea de iguales o diferentes. Los resultados muestran que la habilidad de categorización de variedades entre los participantes fue pobre o deficiente, afectada principalmente por la escasa exposición y experiencia con mayahablantes de otras comunidades. Los límites geográficos, la distinción entre habla urbana/rural y entre la jach maya (la maya pura, antigua, verdadera) y el xe'ek' (la maya mezclada) también se identificaron como una guía de reconocimiento de variedades o zonas dialectales. Los elementos suprasegmentales (entonación, melodía, ritmo, velocidad de la elocución) y el léxico, en general, resultaron los elementos lingüísticos salientes que guiaron la percepción dialectal de los participantes.

Palabras clave: variedades dialectales; percepción dialectal; maya yucateco; supradialecto; México 


\begin{abstract}
From a perceptual dialectology perspective, ability by some participants to categorize this exploratory descriptive study aims to examine the ability of some bilingual MayaSpanish speakers to identify potential varieties of Maya and relate them to a particular geographical region; determine the most salient features used by people to differentiate one variety from another; and identify factors that may affect this dialectal perception. Some tools from the methodology used by Preston (1999), Mase (1999), and Clopper and Pisoni (2004) were adapted in the present study, such as the categorization task using a map, and the same-different task. We found a poor or deficient the different varieties, affected mainly by the scarce exposure and experience with Maya speakers from other communities. The geographic boundaries and the distinction between rural/urban and between Jach Maya (the pure, ancient, true language) and $X e^{\prime} e k^{\prime}$ Maya (the mixed language) were also used by participants as a guide to recognize a variety or dialectal zone. In general, suprasegmental elements (intonation, melody, rhythm, speed) and the lexicon were found to be salient linguistic elements that also guided the dialectal perception of the participants.
\end{abstract}

Keywords: dialectal varieties; dialectal perception; Yucatec Maya; supradialect; Mexico 


\section{Introducción}

Estudios previos sobre percepción dialectal evidencian que los hablantes/oyentes, no especialistas, pueden hacer juicios confiables sobre el lugar de procedencia de un hablante desconocido, sin la necesidad de instrucciones explícitas acerca de qué es lo que debe escuchar o a qué debe poner atención. Esta habilidad perceptual sugiere que los hablantes retienen en su memoria las variedades de su lengua materna y que estas representaciones se desarrollan de manera natural a través de sus experiencias y exposición a su comunidad de habla y al resto (Clopper, 2004: 21). De esta manera, a partir de los trabajos de Niedzielski (1999), Preston (1999), Long y Preston (2002) y Niedzielski y Preston (2003) se ha ampliado el alcance de la dialectología perceptiva o perceptual, pues de tener como objetivo inicial la identificación de las percepciones de no especialistas sobre las diferencias dialectales, se pretende ahora lograr la comprensión de los procesos sociales y cognitivos que guían al ser humano al conformar dichas percepciones. El interés radica, en la actualidad, en comprender cómo los oyentes perciben, codifican y representan en su memoria la variación dialectal.

Las investigaciones más recientes sobre dialectología perceptual se han realizado en países desarrollados, como en Estados Unidos (Fridland \& Bartlett, 2006; Bucholtz, Bermudez, Fung, Vargas \& Edwards, 2008; Preston, 2011, 2013; Evans, 2013); Francia (Kuiper, 2005; Boughton, 2006); Noruega (Røsstad, 2009; Strand, 2012); Austria (Pfrehm, 2010); Alemania (Gessinger, 2010) y el Reino Unido (Hiraga, 2005; Montgomery, 2012; Montgomery \& Stoeckle, 2013), principalmente. Las lenguas más estudiadas, por tanto, son el inglés, el francés, el noruego y el alemán. En México, la investigación en esta disciplina es incipiente, según registros a mi alcance (véase Serrano, 2002, 2009; Morúa \& Serrano, 2004; Erdösová, 2011; Porter, 2015), y ha versado sobre variación dialectal del español.

Con respecto a la lengua maya yucateca, Pfeiler (1985, 1995, 1997, 1999), Briceño (2002), Bastarrachea y Canto (2003) y Pfeiler y Hofling (2006) han discutido la variación dialectal en el maya yucateco y han aportado con ello los primeros materiales sobre percepción de la variación en esta lengua originaria. No obstante, la mayoría de estos estudios no tenían como propósito principal investigar las percepciones de los usuarios de esta lengua sobre la variación dialectal. 
Dada esta situación, es clara la necesidad de realizar estudios sistematizados sobre la percepción dialectal en México; sobre el español y, por lo menos, sobre las principales lenguas amerindias con las que convivimos, ${ }^{1}$ las cuales gozan de una vitalidad considerable. Por tanto, para este estudio nos planteamos como objetivos: examinar la habilidad de los hablantes para identificar posibles variedades de lengua maya y relacionarlas con regiones o zonas geográficas; determinar los rasgos lingüísticos más salientes que usan para distinguir una variedad de otra, e identificar algunos factores extralingüísticos que pueden afectar tal percepción dialectal.

Las preguntas que guiaron esta investigación fueron: ¿Qué nivel (léxico, fonético-fonológico, sintáctico) o rasgos lingüísticos son los más salientes y los que permiten que los hablantes distingan variedades? ¿Qué factores extralingüísticos afectan la percepción de los informantes (edad, sexo, exposición a otras variedades, estereotipos o connotaciones preconcebidas, medios de comunicación)? ¿Qué facilidad o dificultad presentan los informantes para identificar variedades dialectales del maya yucateco?

\section{Perspectiva teórica sobre la percepción dialectal}

En esta sección, presentamos primeramente algunas propuestas teóricas e hipótesis sobre la percepción dialectal, seguidas de varias sugerencias más sobre la variación dialectal en maya yucateco que han realizado estudiosos de esta lengua originaria. Ambas conforman el marco para discutir nuestros hallazgos.

\subsection{Propuestas teóricas e hipótesis sobre la percepción dialectal}

De acuerdo con Preston (2018), la dialectología perceptual es la rama de la lingüística "popular" (folk linguistics) que estudia la distribución regional de las diferentes variedades dialectales a partir de la percepción de los no especialis-

1 La lengua náhuatl cuenta con 1725620 hablantes, lo que la convierte en la lengua indígena con mayor número de hablantes en México, seguida por la lengua maya con 859607 hablantes (Instituto Nacional de Estadística y Geografía, 2015). 
tas (los hablantes populares), y ha considerado desde sus inicios tanto factores sociales como actitudinales (2018: 177; traducción propia). ${ }^{2}$

El estudio de las percepciones lingüísticas resulta relevante en tanto que se indaga la relación entre la cognición, el lenguaje y la cultura. Vargas señala que la percepción es un mecanismo cognoscitivo, el cual posee un carácter

biocultural porque, por un lado, depende de los estímulos físicos y sensaciones involucrados y, por el otro, de la selección y organización de dichos estímulos y sensaciones. Las experiencias sensoriales se interpretan y adquieren significado moldeadas por pautas culturales e ideológicas aprendidas desde la infancia. La selección y la organización de las sensaciones están orientadas a satisfacer las necesidades tanto individuales como colectivas de los seres humanos, mediante la búsqueda de estímulos útiles y de la exclusión de estímulos indeseables en función de la supervivencia y convivencia social, a través de la capacidad para la producción del pensamiento simbólico, que se conforma a partir de estructuras culturales, ideológicas, sociales e históricas que orientan la manera como los grupos sociales se apropian del entorno (Vargas, 1994: 47).

Con el trabajo sistematizado de más de dos décadas, los estudiosos de la dialectología perceptual en el mundo han derivado algunas hipótesis y propuestas teóricas, que se han tratado de comprobar en diversos estudios que integran ya una agenda de investigación. Entre los factores extralingüísticos identificados que afectan la percepción de la variedad dialectal se encuentran la exposición y experiencia con otras variedades (la interacción con medios de comunicación y redes sociales, así como la movilidad y la proximidad física son algunas de sus formas), estereotipos sociales, edad y clase social.

Preston (1999), por ejemplo, afirma que la gente que está más expuesta a otras variedades es más consciente de las diferencias entre las mismas y muestra un grado mayor de percepción dialectal. Esta exposición y experiencia, sin embargo, pueden estar condicionadas, en cierta medida, por un contacto indirecto

2 La cita original es: "Perceptual dialectology (PD) is the branch of folk linguistics that deals with regional distribution of linguistic features from the point of view of nonspecialists (the "folk"), but has, almost from the beginnings, attended to both social and attitudinal factors". 
(no cara a cara) a través de los medios de comunicación y las redes sociales, por ejemplo; o por un contacto directo (cara a cara) a través de la movilidad social y geográfica de los hablantes (cambio de residencia) o por la proximidad física con otras comunidades (visitas a otras zonas o visitantes de estas).

Ahora bien, son los medios de comunicación los que se han considerado elementos clave para la difusión y fortalecimiento de una variedad. Strand (2012), en su estudio del noruego, describe la influencia directa que los medios pueden ejercer en la percepción dialectal de los hablantes. De la misma manera, Hartley (2005) y Fridland y Bartlett (2006) destacan también la importante función de los medios de comunicación en sus estudios. Queda claro así que, a través de dichos medios, los hablantes reciben input de las variedades ahí mostradas, las cuales pueden incluso ser controladas o privilegiadas.

En cuanto a la movilidad social y geográfica, Kerswill y Williams (2002) encontraron que esta movilidad de los participantes desempeña una función importante en la percepción de las variedades dialectales. Clopper y Pisoni (2004), por su parte, identificaron efectos del historial residencial de los hablantes en la tarea de categorización dialectal.

Otra forma de exposición a otras variedades es la proximidad, principalmente física. En este sentido, la cercanía o lejanía de dos o más comunidades de habla afecta significativamente la percepción, de acuerdo con Montgomery (2012) y Montgomery y Stoeckle (2013). Sin embargo, el primero señala que el fenómeno de la proximidad está condicionado por barreras físicas y psicológicas. El influjo de los medios de comunicación puede hacer que una variedad lejana, en términos geográficos, resulte más "cercana" y familiar que otra de alguna comunidad próxima.

Este factor (los medios de comunicación) está fuertemente vinculado con otro: la prominencia cultural de una variedad, pues los medios de comunicación masiva pueden ser el vehículo de difusión de una variedad y hacerla culturalmente prominente (Montgomery, 2012), o pueden ayudar a su mantenimiento o cambio. Pese a esto, una variedad con prominencia cultural no necesariamente es la promovida por los medios, existen variedades que gozan de prestigio por su historia, por su antigüedad o por ser las que se hablan en zonas identificadas como centros culturales, económicos, políticos o sociales importantes. Por su 
parte, Kuiper (2005) destaca el lugar de procedencia de los participantes y de las voces grabadas como un factor relevante en la percepción dialectal.

Demirci y Kleiner (1998), Gessinger (2010) y Pfrehm (2010) identificaron la edad como una variable relevante en la percepción dialectal y en los juicios que los hablantes generan al respecto. Los primeros encontraron que los jóvenes turcos eran, generalmente, más conservadores que los adultos en sus evaluaciones o juicios hacia otras variedades. En cuanto a la producción, Gessinger (2010) encontró que los adultos mayores tienden a usar construcciones dialectales en habla espontánea, mientras que los jóvenes evidencian una combinación de construcciones dialectales y propias del estándar. Pfrehm (2010) mostró que los jóvenes austriacos tenían percepciones más positivas hacia el estándar alemán que los adultos, por ejemplo.

Otro factor social de consideración es la clase social. Hiraga (2005) reportó que sus participantes británicos evaluaron como prestigiosa tanto la llamada pronunciación aceptada (received pronunciation) ${ }^{3}$ de la variedad británica como la pronunciación de la variedad estándar de Estados Unidos. El autor lo atribuye a la conciencia de los hablantes de relacionar estas variedades con determinadas clases sociales.

Preston (2013) también discute la función de la clase social y las ideologías en los juicios de los participantes sobre las diferentes variedades, y sugiere que puede haber una asociación de dialectos regionales con connotaciones preconcebidas. Además de la cuestión de clase, la percepción dialectal puede ser influida por la existencia de generalizaciones asociadas a ciertas regiones. Por ejemplo, en México, es frecuente asociar el fenómeno de la despalatalización (ninia por niña) con hablantes de la Península de Yucatán.

\subsection{La variación dialectal en maya yucateco}

Si bien la investigación sobre la variación dialectal en maya ha sido, hasta cierto punto, de interés entre los mayistas de todo el mundo, los estudios recientes y sistemáticos son escasos en realidad. Estos se han dividido entre los de corte

3 Roach (2009: 4) la describe como el acento que usan los comentaristas y presentadores de la BBC y de la televisión independiente británica. 
meramente lingüístico, concentrados en las diferencias sintácticas, morfológicas, semánticas o léxicas (Po’ot Yah \& Bricker, 1981; Pfeiler, 1995, 1996; Sobrino, 2005), y aquellos basados en las actitudes, opiniones y percepciones de los hablantes (Pfeiler, 1997, 1999; Berkley, 1998; Briceño, 2002; Bastarrachea \& Canto, 2003). De manera incipiente, se están realizando investigaciones que integran ambas posturas, como la de Pfeiler y Hofling (2006). Este último estudio, en particular, ha sido también pionero en conformar un enorme corpus recolectado en numerosas regiones de los tres estados de la Península de Yucatán e incluso en Corozal, Belice.

A la fecha, se han propuesto las siguientes configuraciones dialectales del maya en la Península de Yucatán:

a) Una división primaria entre el oriente y el occidente peninsulares (Edmonson, 1986).

b) División regional en cinco variantes: oriente de Yucatán, centro de Quintana Roo, sur de Yucatán, Camino-Real-Ch'enes de Campeche y la exzona henequenera, con tres variantes: el $x e^{\prime} e k^{\prime}$, que es hablado en la exzona henequenera, sur y centro de Yucatán; el maya cantado de las regiones del Camino Real y Ch'enes de Campeche, y la variante jach maya, hablada en el sur y oriente de Yucatán y centro de Quintana Roo (Briceño, 2002).

c) Cinco regiones dialectales en la península de Yucatán: exzona henequenera del estado de Yucatán; Camino Real en el estado de Campeche; Valladolid y alrededores (oriente de Yucatán); sur del estado de Yucatán y centro de Quintana Roo, y la región de los Ch'enes, Campeche (Bastarrachea \& Canto, 2003).

De manera interesante, Pfeiler y Hofling destacan que la descripción de Briceño (2002) "concuerda en general con la opinión de la comunidad mayahablante quien distingue entre 'la maya pura', antigua, verdadera (jach maya) y 'la maya mezclada' (el xe'ek’) (Pfeiler, 1985, 1997, 1999; Berkley, 1998)” (Pfeiler \& Hofling, 2006: 29).

Con respecto a la distinción de estas variedades, los mismos autores señalan que "en los hablantes del maya yucateco entrevistados existe una 'imagen lingüística' resultante de una comparación con un determinado ideal lingüístico, 
correspondiente a la norma general, o norma supradialectal" (Pfeiler \& Hofling, 2006: 39). De acuerdo con Pfeiler (1985), esta conciencia lingüística existente entre los hablantes sobre la variación y el cambio en maya yucateco se refleja en la percepción de la jach maya y el $x e^{\prime} e k$ ', variedades que se asocian con factores como la región geográfica, la edad de los hablantes y la ausencia o presencia del español.

La hach maya, hablada en el oriente de la península, es definida como "la maya pura", "la maya antigua", "la maya legítima", o "la maya verdadera". Sólo los "mayeros" de avanzada edad la saben hablar (Pfeiler, 1995: 490). El $x e$ 'ek' se presenta como la variedad decadente, se trata de la variedad moderna, del maya mezclado o corrupto por el español y es hablado principalmente por los jóvenes en el occidente y el centro de la península. La segunda es actualmente la variedad hablada por la mayoría de la población yucateca (Pfeiler \& Hofling, 2006: 39).

\section{Método}

Los estudios de dialectología perceptual que se han realizado hasta ahora han seguido dos enfoques: uno centrado en la percepción de la producción en general de los hablantes y otro en la percepción de los rasgos lingüísticos específicos de las variedades en cuestión. Estos últimos son más recientes y se han apoyado en los métodos sofisticados de las ciencias del habla y de la fonética acústica (Preston, 2018).

Preston distingue entre perceptos y conceptos, los primeros son generados por las reacciones de los hablantes a datos lingüísticos reales, los segundos se refieren al mundo conceptual de los hablantes, en ocasiones basado en percepciones previamente recibidas. Por ejemplo, en una tarea como la de identificar en un mapa fronteras dialectales, lo que se suscita en el hablante son conceptos, dado que no se le provee de muestras de habla para escuchar. Por lo contrario, las tareas del enfoque meramente perceptual se enfocan en las reacciones de los hablantes a muestras reales de habla. Así, en la primera tarea, es el consciente el que genera los conceptos, mientras que, en la segunda, los perceptos son generados desde el inconsciente del hablante. Según el mismo autor, la dialec- 
tología perceptual actual integra ambos enfoques, conceptos y perceptos, en sus estudios.

Niedzielski y Preston (2003), al respecto, asumían que la lingüística popular (folk linguistics) se enfocaba en lo consciente, mientras que los estudios de actitudes lingüísticas, en el inconsciente, pero destacan que esta distinción entre los dos campos de estudio, más que llevar a una dicotomía, implicaba un continuo. La investigación en dialectología perceptual ha explorado técnicas para motivar actitudes tanto desde el plano del consciente como desde el inconsciente (como las técnicas del par oculto o matched-guise y las respuestas neurológicas), según el mismo Preston (2018).

Aquí se presenta un estudio exploratorio y descriptivo en el que se adaptaron, con algunas modificaciones, dos tareas de la metodología de Mase (1999), Preston (1999) y Clopper y Pisoni (2004): la tarea de categorización controlada con mapa y la tarea de iguales o diferentes. Dado que, en relación con la distribución dialectal del maya yucateco desde la percepción de los hablantes, no hay estudios hasta ahora, para este estudio exploratorio nos enfocamos en la percepción de la producción general.

La recolección de datos se hizo en Chetumal, Quintana Roo, con informantes originarios de distintos poblados. La muestra, la cual fue por conveniencia, se conformó por 15 informantes: nueve hombres y seis mujeres; 10 eran universitarios y cinco habían estudiado hasta la secundaria; 9 tenían entre 18 y 25 años, y seis, entre 26 y 50. Todos eran bilingües de maya-español, pero para 11 el maya era su lengua materna y para cuatro, el español. Todos eran hijos de padres mayahablantes. En cuanto a su lugar de origen, uno era de Buena Esperanza; uno, de Los Divorciados; uno, de Limones; uno, de Peto; dos, de Valladolid; dos, de José María Morelos, y siete, de Carrillo Puerto. Reconocemos que una limitante en este estudio es la falta de una muestra equilibrada, pues no se logró conformar así por cuestiones de tiempo y disponibilidad de los informantes. Por tratarse de un estudio exploratorio, no obstante, el número de informantes es adecuado.

Para la tarea de categorización controlada con mapa, se realizaron grabaciones de seis mayahablantes originarios de los siguientes poblados: Mama y Peto (Yucatán); Tihosuco, Señor, Uh May y Limones (Quintana Roo). Se consideraron dos hablantes de Yucatán y cuatro de Quintana Roo para indagar si la per- 
cepción dialectal era más notable entre estados y entre poblados distribuidos en el norte, centro y sur de Quintana Roo. Los sujetos grabados fueron cinco hombres y una mujer. Cada uno de los sujetos se grabó relatando en maya la celebración del Hanal Pixan ${ }^{4}$ durante un minuto. Las grabaciones se colocaron en orden aleatorio. Se elaboró también un mapa con los nombres de los poblados de los hablantes ${ }^{5}$ (véase Figura 1), en el cual los informantes tenían que relacionar las voces con las regiones señaladas.

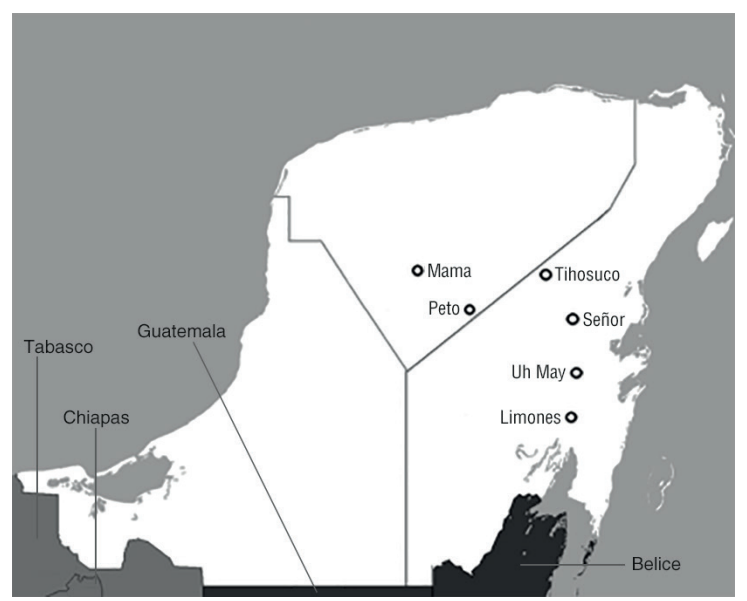

FIGURA 1. Lugar de origen de las voces grabadas

Para la tarea de iguales o diferentes se conformaron pares de estas grabaciones: Limones y Tihosuco; Uh May y Mama; Señor y Peto. El criterio de este emparejamiento era la pertenencia a estados diferentes y, en el caso de Tihosuco, la proximidad a Yucatán.

4 El Hanal Pixan es una celebración realizada en Yucatán, en la que se ofrenda comida y bebida a los fieles difuntos para honrar su memoria y mantener el vínculo entre vivos y muertos (Buenfil, Ramayo \& Rodríguez, 1999). Actualmente, también se celebra en Quintana Roo.

5 Se decidió hacer esta tarea controlada y no libre, como se ha realizado en estudios previos, tras haber efectuado una prueba piloto con tres informantes y observado que tenían muchas dificultades para ubicar en el mapa algún poblado. 
Al final de las tareas, se realizaron preguntas abiertas a los informantes sobre sus antecedentes lingüísticos, su exposición a otras variedades y sobre sus percepciones de la forma de hablar de otras comunidades mayahablantes.

Los datos recogidos se analizaron cuantitativamente; se ofrecen adelante las frecuencias y porcentajes de los resultados. Asimismo, se revisaron las respuestas de las preguntas abiertas y se establecieron algunas categorías de análisis, basadas principalmente en la perspectiva teórica que se detalló en la sección previa.

\section{Resultados y discusión}

Dado que uno de los objetivos planteados en este estudio es examinar la habilidad de los hablantes para identificar y relacionar una posible variedad de lengua maya con una región geográfica, en la Figura 2 se presentan los resultados de la tarea de categorización de las grabaciones.

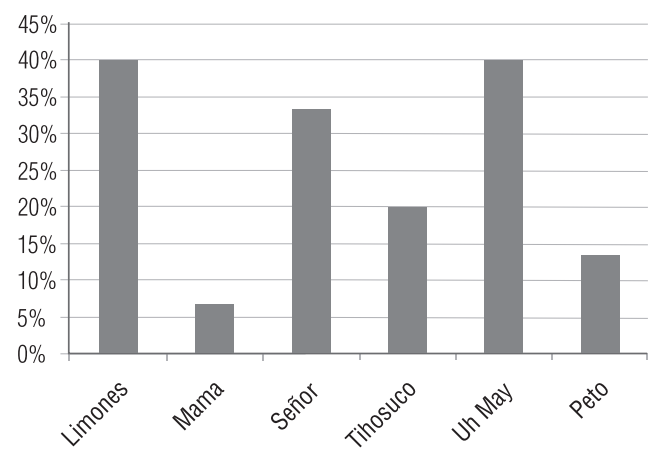

FIGURA 2. Porcentaje de precisión en la tarea de categorización controlada en un mapa

Como se observa, las grabaciones que alcanzaron un mayor porcentaje de asociación con el lugar de origen fueron la de Limones (40\%, 6/15), Uh May $(40 \%, 6 / 15)$ y Señor $(33.3 \%, 5 / 15)$. Las que tuvieron más errores de precisión en la categorización fueron Tihosuco $(20 \%, 3 / 15)$, Peto $(13.3 \%, 2 / 15)$ y Mama $(6.7 \%, 1 / 15)$. Estudios previos, como el de Williams, Garrett y Coupland (1999) y Clopper (2004), reportan un 30\% de precisión en la categorización de 
dialectos. Si bien en nuestros datos tres variedades fueron clasificadas en un porcentaje mayor a $30 \%$, la identificación de las otras tres variables está muy por debajo de esta cifra. Lo que es evidente es que las variedades del centro y sur de Quintana Roo (Limones, Señor y Uh May) fueron mejor percibidas que las de Yucatán (Peto y Mama) o que la muy cercana a este estado (Tihosuco).

Los resultados de esta primera tarea pueden deberse a la limitada exposición y poca experiencia con mayahablantes de otras comunidades, lo cual se evidencia, por ejemplo, en el hecho de que la mayoría de los informantes eran provenientes del centro y sur de Quintana Roo; de lugares muy cercanos a las variedades grabadas que alcanzaron mayores porcentajes de precisión en esta tarea. Es decir, la proximidad geográfica parece ser un factor condicionante de la percepción dialectal, como ya lo señalaba Kuiper (2005) al advertir la importancia del lugar de procedencia de los participantes y de las voces grabadas en la percepción dialectal; esto coincide también con estudios como los de Montgomery (2012) y Montgomery y Stoeckle (2013).

En la segunda tarea, similares o diferentes, los resultados del par Limones-Tihosuco muestran que $80 \%$ de los participantes indicaron que son diferentes; del par Uh May-Mama, 60\% eligió diferentes, y lo mismo ocurrió para el par Señor-Peto. Dado que estos grupos se integraron por una voz del centro o sur de Quintana Roo y una de Yucatán o muy próxima a este estado, se esperaba que las diferencias fueran claramente perceptibles. No obstante, los datos no son contundentes excepto por el caso de Limones-Tihosuco que alcanzó 80\%. Nuevamente, es la variedad de Limones la que parece distinguirse más claramente de las otras variedades.

Esto nos lleva a nuestro segundo objetivo, que es determinar los rasgos lingüísticos más salientes que usan los oyentes para distinguir una variedad de otra. En las entrevistas, casi todos los participantes coincidieron en que los elementos que los guían para distinguir mayahablantes de otras variedades son rasgos suprasegmentales, como la entonación, el ritmo, la melodía y la velocidad de la elocución; y el léxico, principalmente. Se referían a estos aspectos como habla cantadita, habla fluida, habla con alegría y expresión; y modismos, proverbios, expresiones, palabras. Solo un informante dijo que algunos verbos se conjugaban de manera diferente en algunas variedades. 
Estos son algunos comentarios al respecto:

(1) He notado diferencias. Por ejemplo, tengo familiares en Yucatán y pues el tono en el que hablan sí se distingue demasiado... sí, y en vocabulario también. [Informante 2 , mujer]

(2) Es fácil identificar a veces a una persona de otro pueblo por el vocabulario que usa. Te puedes dar cuenta rápido, por ejemplo, en las preposiciones. [Informante 1, hombre]

(3) La entonación de esas personas que hablan el maya más antiguo es como cantadito, más cantadito... Donde se me hace difícil es en Tihosuco y Sabán porque hay unas palabras donde hablan diferente a la maya que mayormente nosotros conocemos. [Informante 7, mujer]

(4) Cuando voy a Valladolid, siento el cambio drástico, ya que tienen cierta alegría y cierta expresión cuando hablan. [Informante 8, hombre]

(5) O la tonalidad porque hay personas que hablan muy lenta la maya y la hacen cantada; entonces también tienes que escucharla bien; si no, no les entiendes. [Informante 12 , mujer]

(6) Sé más o menos de dónde vienen y sé también cuando somos de aquí, de por aquí del sur por la forma en la que hablamos: más despacio, más claro, más pausado también. [Informante 14, hombre]

También, algunos destacan diferencias fonético-fonológicas en el nivel léxico:

(7) Hay unas palabras, por ejemplo, en Yucatán hablan un maya diferente; hay unas palabras que acá en Quintana Roo significan lo mismo, pero se pronuncian diferente. [Informante 4, hombre]

(8) Hay algunos modismos que no suenan igual; cambian un poquito; pero sí les entiendo. [Informante 13, hombre] 
Nótese que los elementos lingüísticos en los que los participantes basan su percepción se limitan a dos niveles (fonético-fonológico y léxico) y las características para describirlos son bastante generales; es decir, estos elementos lingüísticos no son identificados con precisión. Hablan de pronunciación diferente, no suenan igual, el vocabulario es diferente, pero su conciencia lingüística y procesos cognitivos aún no conllevan el notar u observar detalles particulares y abstraerlos.

Las diferencias se establecen, generalmente, entre el maya de Yucatán y el maya de Quintana Roo. Incluso, dos participantes mencionaron el habla de Campeche también como una variedad diferente.

(9) En Campeche hay una especie de maya que no es maya peninsular; es como los tzotziles, tzeltales. Cuando es así, es un dialecto. [Informante 8, hombre]

De esta manera, parece que los participantes en este estudio se guían a partir de los límites geográficos que configuran un estado o una región y que le confieren cierta identidad para distinguir variedades dialectales. Esto, no obstante, no coincide con las configuraciones dialectales propuestas por los estudiosos del maya yucateco (Edmonson, 1986; Briceño, 2002; Bastarrachea \& Canto, 2003).

Además de este elemento geográfico que guía la percepción dialectal de estos participantes, resultó abrumadora la distinción entre la jach maya (la maya pura, antigua, verdadera) y el $x e^{\prime} e k$ ' (la maya mezclada), que ya Pfeiler desde 1985 había identificado. La misma autora señala que estas variedades se asocian con factores como la región geográfica, la edad de los hablantes y la ausencia o presencia del español. Abajo citamos comentarios que ilustran la importancia del factor región geográfica en esta distinción:

(10) El otro me suena más a los que hablan de Tihosuco, ya que mantienen un poquito más lo que es la raíz de la maya. [Informante 1, hombre]

(11) Al principio, quizás con un pueblo que está cerca del mío que se llama La Noria, porque ellos están allá como a $10 \mathrm{~km}$ metidos en el monte y las primeras veces que iba, pues los niños se reían de mí porque la pronunciación la hacía diferente y se escuchaba otra cosa. [Informante 9, mujer] 
(12) La diferencia es entre los que están en el monte y los que se van a las ciudades. En el monte sí se habla un maya puro, antiguo, original. [Informante 7, hombre]

(13) Podría ser que encuentre alguna diferencia en la forma de hablar maya de una persona si me voy a Mérida o al norte de Yucatán; si estoy en el sur de Yucatán, es casi lo mismo (que en Quintana Roo). [Informante 11, hombre]

En términos geográficos, por una parte, estos participantes asocian la jach maya con las comunidades rurales y el $x e^{\prime} e k^{\prime}$ con las urbanas. ${ }^{6}$ Por otra parte, las personas de Quintana Roo tienden a percibir el maya del centro y norte de Yucatán (aunque algunos incluso consideran los pueblos de Quintana Roo cercanos a Yucatán) como las variedades más puras y más originales, donde se habla la raíz del maya. Los participantes provenientes de Yucatán, por su parte, notan diferencias considerando el contraste urbano/rural.

Esta cuestión geográfica, sin embargo, parece interactuar intensamente con el factor ausencia o presencia del español para la clasificación de estas variedades. Los participantes señalaron lo siguiente:

(14) En el norte hablan más el maya puro, las pequeñas comunidades porque no están muy urbanizadas, los que no cuentan como, por ejemplo, con tele, los que no están tan influenciados con el español. [Informante 6, hombre]

(15) Yucatán se diferencia porque hablan en un diferente tono de voz y utilizan palabras específicas; ellos no hacen muchas prestaciones del español y los de Quintana Roo sí hacen muchas prestaciones... Quintana Roo tiene un maya más nuevo, utilizan más palabras prestadas [Informante 13, hombre].

(16) Creo que no hay alguna diferencia de personas de otras comunidades al hablar maya, la única diferencia de las personas de otras comunidades y la nuestra, es que la nuestra ya no se habla como debe de ser, ya está españolizada [Informante 4, mujer].

6 Danielson (2014) señala que, con frecuencia, la variación regional es evidente en los contrastes que los hablantes identifican entre el habla de áreas rurales y urbanas. 
(17) La verdad tampoco creo que sean del mismo pueblo. El segundo habló como de los antiguos mayas y el que habló primero es como un chavo que mete español, por sus palabras. El primero habla como los que hablan ahorita y el segundo como los antiguos, como se hablaba maya [Informante 11, hombre].

(18) Los de Yucatán son los que hablan maya así, así sin meterle nada de español. En Quintana Roo, hay personas que le meten y nada más alargan la palabra para que digan que es en maya aunque la palabra la estén diciendo en español [Informante 12 , hombre].

La edad también resultó un factor relevante en la percepción de estas dos variedades:

(19) La mayoría de la gente habla en maya, no en español, entonces la maya de ellos es más antigua de lo que aquí nosotros conocemos, la gente no lo mezcla con el español. Al menos la gente mayor no; la gente de 60, 65 años hablan la maya sin español. Cuando son mayores de edad [refiriéndose a los 18 años] es el mismo, pero a partir de los 40, 35 años ya lo hablan diferente. [Informante 7, mujer, grupo etario II (entre 26 y 50 años)]

(20) A veces puedo identificar a las personas que hablan maya y saber de dónde son; hay diferencias en vocabulario, por ejemplo. También se puede identificar la edad si usan un maya antiguo o si usan un maya con español y así... [Informante 6, hombre, grupo etario II (entre 26 y 50 años)]

(21) Los más jóvenes usan más palabras del español, lo mezclan; los mayores, no. [Informante 8 , hombre, grupo etario III (más de 50 años)]

Estos hallazgos aportan evidencia a la propuesta de clasificación de Pfeiler en sus distintos trabajos, pues contribuyen con datos sobre la percepción de personas del centro y sur de Quintana Roo. Los resultados coinciden con los hallazgos de Pfeiler y Hofling (2006: 39) respecto a la distinción que hacen los hablantes de la jach maya y el xe'ek'; entre una lengua pura y legítima hablada 
por la gente adulta mayor y una variedad "impura" (influida por el español) hablada por los jóvenes.

Sin embargo, es importante observar también las actitudes que acompañan estas percepciones, ya que es patente la diferencia entre jóvenes y adultos mayores sobre estas dos variedades. Aunque ninguno de los participantes usó los términos jach maya ni $x e^{\prime} e k$ ' para referirse a estas variedades, los jóvenes usaron maya antigua, maya raíz, maya original o simplemente maya para la primera; y hablaban de una mezcla, maya españolizada, maya mezclada, influenciada por el español, con prestaciones, entre otros, para la segunda. Dos personas mayores (una en sus 40 y otra en sus 50) se refirieron a la variedad xe'ek' como maya corrupta, lengua prostituida; y al hablar de la jach maya, usaron original, auténtica. Su actitud era negativa hacia la primera y positiva hacia la segunda. Los jóvenes mostraron actitudes positivas hacia ambas variedades, aunque parecen considerar la jach maya más prestigiosa. Ambos grupos etarios parecen percibir esta última variedad como un ideal lingüístico, lo que concuerda muy bien con lo afirmado por Pfeiler y Hofling (2006: 39): "En los hablantes del maya yucateco entrevistados existe una 'imagen lingüística' resultante de una comparación con un determinado ideal lingüístico, correspondiente a la norma general, o norma supradialectal".

\section{A manera de conclusión}

Con este estudio exploratorio, se intenta contribuir a la incipiente investigación sobre la percepción dialectal en México, en general, y a la percepción dialectal de la lengua maya, en particular. Este tipo de investigaciones, basadas en las percepciones de hablantes no especialistas, puede motivar y ser fuente de futuras investigaciones empíricas, ya que dichas percepciones posiblemente tengan un impacto en la producción de la lengua y afectar la variación y el cambio lingüísticos (Preston, 1989: 2).

Los resultados de esta investigación muestran una habilidad de categorización de variedades, en cierta medida, pobre o deficiente por parte de los participantes, aunque en algunos casos el grado fue similar al de estudios previos. Esta percepción puede estar afectada principalmente por la exposición y la experiencia con mayahablantes de otras comunidades, las cuales están condicio- 
nadas por la proximidad, el lugar de origen y la movibilidad social y geográfica de los participantes, así como por la prominencia cultural de los lugares de origen y las variedades en cuestión.

Por otra parte, los elementos lingüísticos salientes que se identificaron como los que guían la percepción dialectal de los participantes son los rasgos suprasegmentales (entonación, melodía, ritmo, velocidad de la elocución) y el léxico, en general.

Otros aspectos extralingüísticos en los que se basan los participantes son los límites geográficos que configuran un estado o una región y que le confieren cierta identidad. Así, hablan del maya de Yucatán, de Quintana Roo y de Campeche. No obstante, también distinguen el maya del sur de Quintana Roo y el del centro. También fue común la distinción urbana/rural en la percepción de las variedades.

Del mismo modo, fue evidente la distinción que hacen los hablantes entre la jach maya (la maya pura, antigua, verdadera) y el $x e$ ' $e k$ ' (la maya mezclada); variedades que se asociaron con la región geográfica, la edad de los hablantes y la ausencia o presencia del español. Las actitudes que se observaron de manera tangencial en este estudio muestran diferencias entre los jóvenes y adultos mayores hacia estas dos variedades, aunque ambos grupos parecen considerar la jach maya como un supradialecto o una supranorma.

Dadas las limitaciones de este estudio, se sugiere realizar futuras investigaciones con muestras más amplias y más equilibradas en relación con factores sociales y con el lugar de origen de los participantes y de las voces grabadas.

\section{Referencias}

Bastarrachea Manzano, Ramón Juan, \& Canto Rosado, Jorge Manuel (Coords.) (2003). Diccionario maya popular: maya-español/español-maya. Mérida: Instituto de Cultura de Yucatán.

BERKLEy, ANTHONy RoBert (1998). Remembrance and revitalization: The archive of pure Maya (Tesis de doctorado). University of Chicago, Chicago.

Boughton, ZoË (2006). When perception isn't reality: Accent identification and perceptual dialectology in French. Journal of French Language Studies, 16(3), 277-304. 
Briceño Chel, Fidencio (2002). Lengua e identidad entre los mayas de la península de Yucatán. En Los investigadores de la cultura maya (vol. 10, tomo 2, pp. 370-379). Campeche: Universidad Autónoma de Campeche.

Bucholtz, Mary; Bermudez, Nancy; Fung, Victor; Vargas, Rosalva, \& Edwards, Lisa (2008). The normative north and the stigmatized south: Ideology and methodology in the perceptual dialectology of California. Journal of English Linguistics, 36(1), 62-87.

Buenfil, Valerio; Ramayo, Teresa, \& Rodríguez, Juan Carlos (1999). Hanal Pixan, alimento de las ánimas. Mérida: Instituto de Cultura de Yucatán.

Clopper, Cynthia (2004). Linguistic experience and the perceptual classification of dialect variation (Tesis de doctorado). Indiana University, Bloomington.

Clopper, Cynthia, \& Pisoni, David (2004). Homebodies and army brats: Some effects of early linguistic experience and residential history on dialect categorization. Language Variation and Change, 16(1), 31-48.

Danielson, Madalyn (2014). 'Bogans' and boundaries: A perceptual dialectology of Australian English (Tesis de licenciatura). New York University, Nueva York.

Demirci, Mahide, \& Kleiner, Brian (1998). Gender and age-based variation in the perception of Turkish dialects. Language Awareness, 7(4), 206-222.

Edmonson, Munro (1986). Heaven born Merida and its destiny: The book of Chilam Balam of Chumayel. Austin: University of Texas Press.

Erdösová, Zuzana (2011). El español de México en los ojos de sus hablantes: un estudio desde la sociolingüística y la dialectología perceptiva. Lengua y Voz, 1, 57-81.

Evans, Betsy (2013). Seattle to Spokane: Mapping perceptions of English in Washington. Journal of English Linguistics, 41(3), 268-291.

Fridland, Valerie, \& Bartlett, Kathryn (2006). Correctness, pleasantness, and degree of difference ratings across regions. American Speech, 8(4), 358-386.

Gessinger, JoAchim (2010). Language variation, language change and perceptual dialectology. Multilingua: Journal of Cross-Cultural and Interlanguage Communication, 29(3-4), 361-383.

Hartley, Laura (2005). The consequences of conflicting stereotypes: Bostonian perceptions of U.S. dialects. American Speech, 80(4), 388-405.

Hiraga, Yuko (2005). British attitudes towards six varieties of English in the USA and Britain. World Englishes, 24(3), 289-308.

Instituto Nacional de Estadística y Geografía (INEGi) (2015). Encuesta intercensal 2015: lenguas indígenas en México y hablantes (de 3 años y más al 2015). Recuperado de http:// cuentame.inegi.org.mx/hipertexto/todas_lenguas.htm 
Kerswill, Paul, \& Williams, Ann (2002). Dialect recognition and speech community focusing in new and old towns in England: The effects of dialect levelling, demographic and social networks. En Daniel Long \& Dennis Preston (Eds.), Handbook of perceptual dialectology (vol. 2, pp. 173-204). Ámsterdam: John Benjamins.

Kuiper, Lawrence (2005). Perception is reality: Parisian and Provençal perceptions of regional varieties of French. Journal of Sociolinguistics, 9(1), 28-52.

Long, Daniel, \& Preston, Dennis (Eds.) (2002). Handbook of perceptual dialectology. Vol. 2. Ámsterdam: Johns Benjamins.

Mase, Yoshio (1999). Dialect consciousness and dialect divisions. Examples in the Nagano-Gifu boundary. En Dennis Preston (Ed.), Handbook of perceptual dialectology (vol. 1, pp. 71-99). Ámsterdam: John Benjamins.

Montgomery, Chris (2012). The effect of proximity in perceptual dialectology. Journal of Sociolinguistics, 16(5), 638-668.

Montgomery, Chris, \& Stoeckle, Phillipp (2013). Geographical information systems and perceptual dialectology: A method for processing draw-a-map data. Journal of Linguistic Geography, 1(1), 52-85.

Morúa, Carmen, \& Serrano, Julio (2004). Dos mil kilómetros de por medio: dialectología perceptual contrastiva del español de México. En Carmen Morúa Leyva \& Rosa María Ortiz Ciscomani (Eds.), VII Encuentro Internacional de Lingüística en el Noroeste: Memorias (tomo II, pp. 253-276). Hermosillo: Universidad de Sonora.

NiEDZIELSKI, NANCY (1999). The effect of social information on the perception of sociolinguistic variables. Journal of Language and Social Psychology, 18(1), 62-85.

Niedzielski, Nancy, \& Preston, Dennis (2003). Folk Linguistics. Berlín: De Gruyter Mouton.

Pfeiler, Barbara (1985). Yucatán: Das Volk und seine Sprache. Zwei Fallstudien zur Bilinguismussituation (Tesis de doctorado). Universität Wien, Viena.

Pfeiler, Barbara (1995). Variación fonológica en el maya yucateco. En Ramón Arzápalo \& Yolanda Lastra (Eds.), Vitalidad e influencia de las lenguas indígenas en Latinoamérica (pp. 488-497). México: Universidad Nacional Autónoma de México.

Pfeiler, Barbara (1996). Yan difereensia waye' yéetel máaya yukatáan (un estudio dialectal). En Ueli Hostettler (Ed.), Los mayas de Quintana Roo: investigaciones antropológicas recientes (pp. 7-11). Berna: Universität Bern.

Pfeiler, BArbara (1997). El xe'ek' y la hach maya: cambio y futuro del maya ante la modernidad cultural en Yucatán. En Andreas Koechert \& Thomas Stolz (Eds.), Convergencia e individua- 
lidad: las lenguas mayas entre hispanización e indigenismo (pp. 125-140). Hannover: Verlag für Ethnologie.

Pfeiler, Barbara (1999). Situación sociolingüística. En Ana García de Fuentes \& Alfredo Alonzo Aguilar, Juan Jiménez Osornio, Roger Orellana Lanza y Enrique Urzaiz Lares (Coords.), Atlas de procesos territoriales de Yucatán, México (pp. 269-299). Mérida: Facultad de Arquitectura, Universidad Autónoma de Yucatán.

Pfeiler, Barbara, \& Hofling, Andrew (2006). Apuntes sobre la variación dialectal en el maya yucateco. Península, 1(1), 27-44.

Pfrehm, James (2010). The role of age in Austrians' perceptions of the frequency of use and likeability of lexical teutonisms and austriacisms. Folia Linguistica, 44(2), 439-470.

Po'ot Yah, Eleuterio, \& Bricker, Victoria (1981). Yucatec Maya verbs (Hocabá dialect). Nueva Orleans: Tulane University.

Porter, Newell Douglas (2015). La dialectología en México: hacia un enfoque perceptual (Tesis de maestría). Brigham Young University, Provo.

Preston, Dennis (1989). Perceptual dialectology: Non-linguists' view of aerial linguistics. Dordrecht: Foris.

Preston, Dennis (1999). Handbook of perceptual dialectology (Vol. 1). Ámsterdam: John Benjamins.

Preston, Dennis (2010). Perceptual dialectology in the 21st century. En Christina Ada Anders, Markus Hundt \& Alex Lasch (Eds.), "Perceptual dialectology” Neue Wege der Dialektologie (pp. 1-30). Berlín: De Gruyter Mouton.

Preston, Dennis (2011). Methods in (applied) folk linguistics: Getting into the minds of the folk. AILA Review, 24, 15-39.

Preston, Dennis (2013). The influence of regard on language variation and change. Journal of Pragmatics, 52, 93-104.

Preston, Dennis (2018). Perceptual dialectology. En Charles Boberg, John Nerbonne \& Dominic Watt (Eds.), The handbook of dialectology (pp. 177-203). Oxford: Wiley Blackwell.

Roach, Peter (2009). English phonetics and phonology (4 ed.). Cambridge: Cambridge University Press.

RøSSTAD, Rune (2009). Foundations of language perceptions and the role of external factors: A Norwegian case. Language Awareness, 18(2), 96-112.

Serrano, Julio (2002). ¿Cuántos dialectos del español existen en México? Ensayo de dialectología perceptual. Recuperado de https://www.academia.edu/2964549/_Cu\%C3\%A1ntos_ 
dialectos_del_espa\%C3\%B1ol_existen_en_M\%C3\%A9xico_Un_ensayo_de_dialectolog\%C3\%ADa_perceptual

Serrano, Julio (2009). ¿Existe el noroeste mexicano como zona dialectal? Un acercamiento perceptual. En Everardo Mendoza Guerrero, Maritza López Berríos \& Ilda Elizabeth Moreno Rojas (Coords.), Lengua, literatura y región (pp. 107-130). Culiacán: Universidad Autónoma de Sinaloa.

Sobrino Gómez, CARlos MARTín (2005). El fenómeno de elisión y su variación en el maya yucateco (Tesis de licenciatura). Universidad Autónoma de Yucatán, Mérida.

Strand, Thea R. (2012). Winning the dialect popularity contest: Mass-mediated language ideologies and local responses in rural Valdres, Norway. Linguistic Anthropology, 22(1), 23-43.

Vargas Melgarejo, Luz María (1994). Sobre el concepto de percepción. Alteridades, 8, 47-53.

Williams, Angie; Garrett, Peter, \& Coupland, Nikolas (1999). Dialect recognition. En Dennis R. Preston (Ed.), Handbook of perceptual dialectology (vol. 1, pp. 345-358). Ámsterdam: John Benjamins. 
